\title{
RESULTADOS DE LA APLICACIÓN DE LA METODOLOGÍA DE AUTOAPRENDIZAJE DEL MÉTODO DE LOS ELEMENTOS FINITOS A CASOS DE TRANSFERENCIA DE CALOR
}

\section{RESULTS OF THE APPLICATIONS OF METHODOLOGY IN THE SELF-LEARNING METHOD OF THE FINITE ELEMENTS TO CASES OF HEATING TRANFERENCES}

\author{
Abraham Farias F. ${ }^{1} \quad$ Gonzalo Salinas S. ${ }^{1}$ \\ Recibido el 21 de marzo de 2005, aceptado el 21 de septiembre de 2005 \\ Received: March 21, 2005 Accepted: September 21, 2005
}

\begin{abstract}
RESUMEN
Este trabajo muestra la metodología y los resultados alcanzados por un estudiante de ingeniería de ejecución en mecánica de rendimiento académico sólo regular, al desarrollar un proceso de autoaprendizaje del método de los elementos finitos aplicados a la problemática de transferencia de calor, utilizando un software comercial. Los resultados alcanzados permiten concluir que el uso de esta metodología fue la adecuada y permitió al sujeto de estudio alcanzar un manejo adecuado del software, satisfactorio dentro de los criterios de evaluación del tipo de dominio, los que demostraron que había alcanzado las competencias necesarias para solucionar un conjunto importante de problemas típicos que involucran fenómenos de transferencia de calor.
\end{abstract}

Palabras clave: Metodología, elementos finitos, transferencia de calor, autoaprendizaje, software FEM.

\section{ABSTRACT}

This paper illustrates the procedures used by an undergraduate engineering student of just mediocre academic standing, when developing a self-learning process using a commercial finite element method package for heat transfer problems. The results show that the student's methodology was correct and allowed her/him to reach a satisfactory understanding of the software according to the criteria used in the application domain. We conclude that this methodology allows the students to achieve the required capabilities for resolving a significant set of heat transfer problems.

Keywords: Procedures, finites elements, heat transfer, self-learning, software FEM.

\section{INTRODUCCIÓN}

Las tendencias modernas en educación indican que el estudiante, en general, durante su vida académica debe adquirir la capacidad de desarrollar competencias por sobre la mera asimilación de los conocimientos ya existentes. Esto conduce a que muchas de las técnicas de enseñanza actualmente utilizadas estén cuestionadas, lo que se extiende al caso de la enseñanza en ingeniería, donde tradicionalmente se ha supuesto que la técnica de aprendizaje se basa en un enfoque de enseñanza frontal, la que no necesariamente conduce a resolver los problemas de aprendizaje y la adquisición de destrezas $\mathrm{y}$ habilidades por parte de los alumnos [1], [2].

La forma tradicional de enseñanza de la ingeniería ha conducido que una parte importante de las cohortes de estudiantes presenten un bajo rendimiento académico, el que se manifiesta a través de bajas calificaciones, continuas reprobaciones, una larga permanencia en la carrera y la pérdida, ya sea temporal o total, de calidad de alumno regular. Esto conduce necesariamente a plantear la interrogante: ¿Reflejan esos índices la verdadera capacidad de aprender, o mejor dicho, adquirir y demostrar competencia por parte de este tipo de estudiantes? [3].

\footnotetext{
${ }^{1}$ Universidad de Talca, Facultad de Ingeniería, Departamento de Plantas y Equipos Industriales, Camino Los Niches km. 1-Curicó, Chile, afarias@utalca.cl,gsalinas@utalca.cl
} 
En búsqueda de una primera aproximación, es que se planteó este trabajo, que presenta el desarrollo y los resultados alcanzados al emplearse una alternativa al enfoque tradicional del proceso enseñanza-aprendizaje -evaluación, representado por la clase frontal y los roles tradicionales de profesor y alumno, por una estrategia de autoaprendizaje, donde al estudiante se le colocan ciertas metas, sin que se le indique la manera de alcanzarlas, pero presentándole alternativas e impulsarlo a que él mismo, a través del autoaprendizaje, pueda adquirir las competencias necesarias que demanda el adquirir una determinada destreza. En este contexto, profesor debe cambiar su rol, para transformarse en un facilitador del conocimiento, de manera que le permita al aprendiz compartir sus experiencias emocionales frente al desafío que constituye la adquisición de nuevos conocimientos y competencias [2], [3].

Con este fin se escogió un sujeto de estudio, con las características ya indicada y se diseñó una metodología para el proceso de enseñanza-aprendizaje-evaluación basada en el autoaprendizaje, de manera que pudiera construir su propio conocimiento de una determinada disciplina o técnica de la ingeniería, que presenta cierta complejidad y que adicionalmente fuera desconocida inicialmente para él. Esto condujo, paralelamente, al desarrollo de estrategias de seguimiento y evaluación de los grados de dominio y competencias alcanzados por el estudiante en las distintas etapas del trabajo [3], [4].

En términos específicos, se escogió la temática relativa a la aplicación del método de los elementos finitos, por medio de un software comercial, para la resolución de diversos problemas de transferencia de calor.

La razón de seleccionar esta aplicación se basó en el hecho que el método de los elementos finitos es una herramienta que está adquiriendo una gran importancia en la ingeniería mecánica, ya que permite predecir el comportamiento de diferentes fenómenos y así generar soluciones a diversos problemas, tales como: el análisis de tensiones, casos de transferencia de calor y flujo de fluidos, así como muchas otras aplicaciones [5].

Por otro lado, ésta es una herramienta especializada y de cierta complejidad, por lo que requiere de un esfuerzo importante para comprender la teoría en la cual se sustenta y realizar sus aplicaciones. A lo que se suma el hecho que en casi todos los currículum de pregrado de estudiantes de ingeniería de ejecución en mecánica no se contemplan cursos formales de elementos finitos, si bien, eventualmente, se estudia el método de las diferencias finitas, ya sea en forma analítica o gráfica.
La metodología empleada consistió en que el estudiante solucionara un conjunto de problemas de transferencia de calor, extraídos de la literatura que normalmente es utilizada en la enseñanza de esta área. Estos problemas se encuentran resueltos mediante técnicas analíticas.

El hecho de utilizar problemas resueltos permite al alumno no sólo conocer de antemano la solución para validar los resultados, sino que le permite, además, adquirir seguridad, desde el punto de vista psicológico, en la exactitud de sus resultados. A la vez que le permite construir la base de conocimientos y perfeccionar sus habilidades en el uso de esta herramienta y aplicarla con seguridad en situaciones más complejas [3].

Respecto de la perspectiva docente, aparece el problema de determinar el grado de avance en el aprendizaje que presenta el alumno, así como el extrapolar los resultados alcanzados con estudiantes de similares características.

Finalmente, cabe consignar que el estudiante presentó un buen grado de dominio al término del proceso, demostrando que había adquirido las competencias necesarias para aplicar adecuadamente este método a diversas situaciones de ingeniería, sin que requiera de un curso formal del método ni de la fundamentación teórica de éste.

\section{DESARROLLO}

En esta sección, se presentan la metodología empleada y los resultados alcanzados al desarrollar este estudio en el Departamento de Tecnologías Industriales de la Universidad de Talca, que presta servicios académicos y docentes a la carrera de Ingeniería de Ejecución en Mecánica.

En términos formales, el ámbito en que se desarrolló el estudio correspondió al incremento de la memoria de título de un estudiante de esta carrera, cuyo perfil corresponde a un estudiante con bajo rendimiento académico, el que se detallará posteriormente.

En cuanto al proceso de seguimiento del grado de avance del estudiante y del efecto que en él tuvieron las técnicas pedagógicas empleadas, fue desarrollado desde dos ópticas. Donde la primera correspondió al docente en contacto directo con el estudiante, mientras que la segunda correspondió a un observador docente externo, quien registraba las actividades del docente directo como del estudiante y avances en el proceso de aprendizaje. 
Las competencias esperadas al finalizar este proceso fueron:

- Comprender los fundamentos conceptuales básicos del método de los elementos finitos.

- Lograr un dominio básico del uso del software.

- Desarrollar una estrategia para la forma de abordar diversos problemas utilizando este método.

- Alcanzar las destrezas necesarias para modelar adecuadamente un determinado problema, tanto en su geometría, selección de tipos de elementos, propiedades del material, restricciones, cargas, mallado, etc.

- Desarrollar su aplicación computacional, lo que implica obtener una solución.

- Interpretar y analizar adecuadamente los resultados obtenidos.

El perfil del estudiante que sirvió de sujeto de estudio, corresponde a un alumno 24 años de edad y que en términos académicos se definió como un estudiante de bajo rendimiento académico.

Esta definición de bajo rendimiento académico se basó en su historial académico como alumno de la carrera de ingeniería de ejecución en mecánica, la que tiene una duración normal de ocho semestres académicos, siendo el primer año de carácter anual, lo que implica que la carrera debería ser completada en un período de cuatro años.

El sujeto de estudio requirió de un total de trece (13) semestres académicos para cumplir con el programa de asignaturas regulares, lo que implicó que el período de permanencia en la carrera fuera de un $67,5 \%$ mayor al período normal. Presentando en esta etapa un total de dos (2) eliminaciones de la carrera debido a su bajo rendimiento académico.

La siguiente lista muestra el comportamiento académico en términos de sus calificaciones promediadas por asignaturas, considerando que la escala de calificaciones es de 1 a 7, con el valor 4 como comprensión mínima de la temática necesaria para la aprobación de la asignatura. De acuerdo a estos parámetros la situación del sujeto de estudio es la siguiente:

- Promedio total asignaturas cursadas: 4,4

- Promedio asignaturas aprobadas: 4,9

Su historial indica que debió cursar 13 asignaturas por segunda vez, lo que implica un $34 \%$ del total, a la vez que debió cursar una asignatura por tercera vez.
Las figuras siguientes muestran en forma de gráficos el comportamiento académico que presentó durante el desarrollo de sus estudios.

El gráfico 1 presenta de manera conjunta el comportamiento académico, en términos de la cantidad de asignaturas cursadas y las reprobadas que cursó en un determinado semestre.

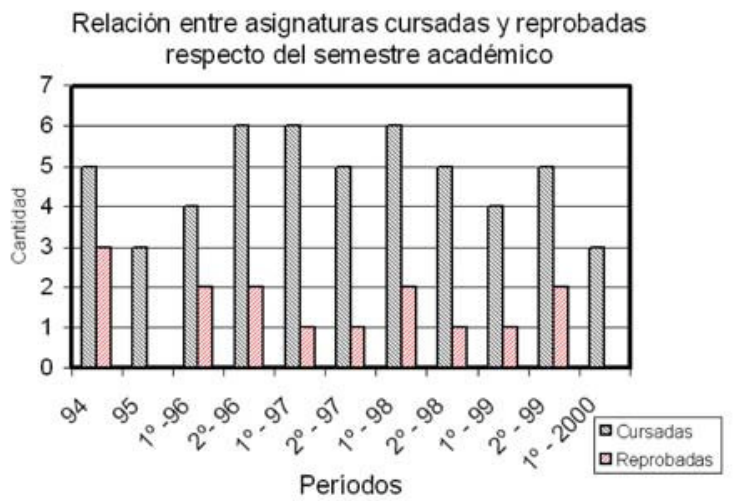

Gráfico 1 Comportamiento académico en términos de aprobaciones y reprobaciones.

El gráfico 2 muestra el comportamiento, en términos porcentuales, de las asignaturas reprobadas durante cada período académico. Como se puede apreciar, el perfil del sujeto de estudio corresponde a lo que comúnmente se considera como un alumno de bajo rendimiento académico, especialmente en los primeros años y que posteriormente se transformó en un rendimiento irregular.

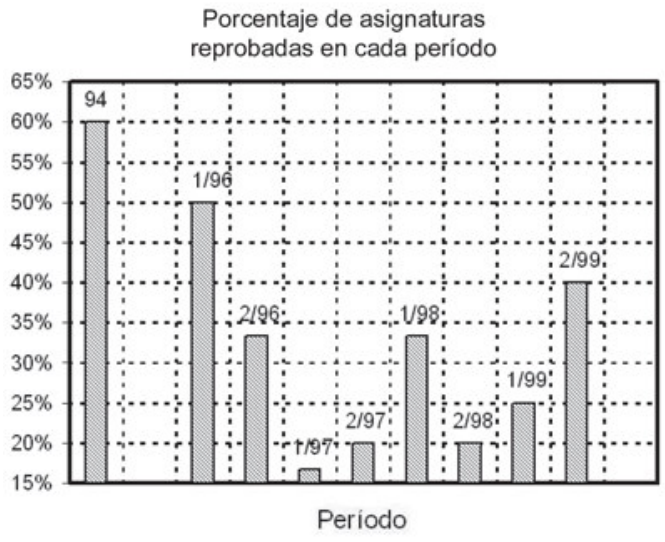

Gráfico 2 Comportamiento académico en términos porcentuales de reprobaciones.

El gráfico 3 muestra, a su vez, el promedio de calificaciones alcanzado por el estudiante en las asignaturas que cursó por primera vez en cada período. 


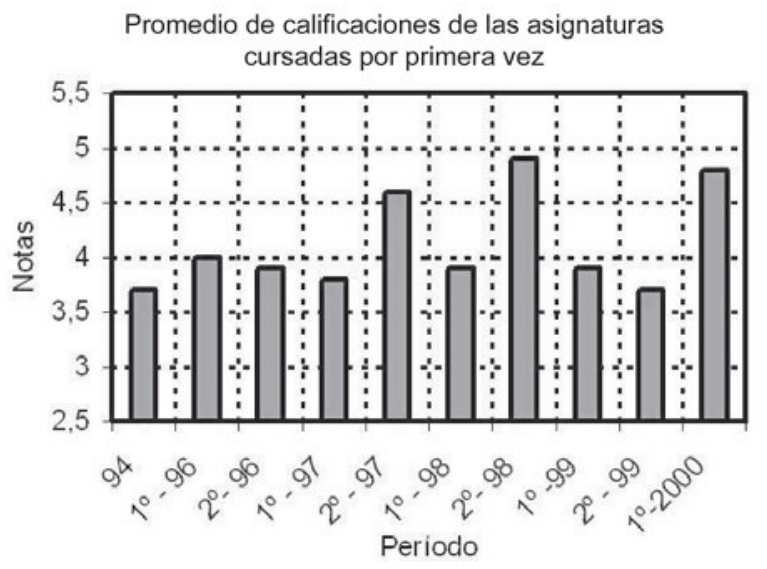

Gráfico 3 Comportamiento académico en términos de calificaciones.

\section{ASPECTOS METODOLÓGICOS}

La metodología empleada para el proceso de enseñanzaaprendizaje-evaluación y su correspondiente organización se estructuraron sobre el objetivo de que el estudiante alcanzara ciertas capacidades o dominios, independiente del tiempo que requiera en alcanzarlos, por lo tanto, su evaluación no tuvo la connotación de obligatoriedad de adquirirlos en un determinado tiempo.

Las etapas que comprendió la metodología empleada fueron estructuradas de la siguiente manera:

- Exposiciones por parte del profesor de los fundamentos teóricos del método de los elementos finitos.

- Un conjunto de sesiones de familiarización con los comandos básicos del software.

- Una revisión bibliográfica, por parte del alumno, sobre los aspectos relevantes del tema, mediante la revisión de los manuales y los tutoriales que en diversas páginas Web se encuentran, en torno al uso del software.

- Interacción elemental con el software para conocer su ambiente y sus procedimientos.

- Desarrollo de ejercicios simples, a fin de adquirir las destrezas y la confianza en los procedimientos ejecutados.

- Evaluaciones del tipo de dominio para determinar el grado de aprendizaje alcanzado por el estudiante.

- Control del estado de avance del proceso de enseñanza-aprendizaje-evaluación por parte del evaluador externo.

- Selección de los problemas de mayor complejidad por parte del profesor y el evaluador externo, cuya solución por parte del estudiante permitirá determinar las competencias alcanzadas.
- Desarrollo de controles periódicos del grado de avance de estudiante por parte del profesor.

- Evaluación del grado de dominio alcanzado por el estudiante por parte del evaluador externo.

- Elaboración de un documento que resuma el trabajo realizado, en la forma de tutorial para autoaprendizaje de nuevos alumnos.

- Evaluación y control final del trabajo por una comisión ad hoc, integrada por el profesor, el evaluador externo y un par.

\section{ALCANCES DEL TRABAJO}

Desarrollada la etapa de introducción con el software, se plantearon a modo de evaluación cinco problemas típicos de transferencia de calor, que fueron extraídos de textos que forman parte de la bibliografía clásica para enseñanza de esta área, los que poseen diferentes grados de dificultad y abordan diferentes temáticas.

Estos problemas se encuentran desarrollados mediante distintas técnicas de soluciones (analíticas o numéricas), lo que permitió en todo momento al estudiante validar los resultados alcanzados por él, con los reportados por la literatura. Los problemas escogidos se indican en la bibliografía.

Esquemáticamente, los casos analizados, ordenados de acuerdo a su complejidad relativa, fueron:

1. Conducción de calor, unidimensional, estacionaria, sin generación interna de calor, con conductividad térmica constante, en un cuerpo prismático.

Las condiciones de borde del cuerpo corresponden a convección [5].

2. Conducción de calor, unidimensional, estacionaria, con generación interna de calor, con conductividad térmica constante, en un cuerpo cilíndrico.

Las condiciones de borde del cuerpo corresponden al campo de temperaturas exteriores conocidas [6].

3. Conducción de calor unidimensional, estacionaria, sin generación interna de calor, con conductividad térmica constante, a través de aletas de sección rectangular del tipo larga, con propiedades del fluido constantes [7].

4. Conducción de calor, bidimensional, estacionaria, sin generación interna de calor, con conductividad térmica constante, en cuerpo prismático.

Las condiciones de borde del cuerpo son de los tres tipos definidos, esto es, campo de temperaturas conocido para determinadas posiciones, aislación perfecta para determinadas posiciones y convección y/o radiación para determinadas posiciones [6]. 
5. Conducción de calor, tridimensional, transiente, sin generación interna de calor, con conductividad térmica constante, en cuerpo cilíndrico.

Las condiciones de borde del cuerpo corresponden a convección [5].

\section{DESARROLLO DE LOS PROBLEMAS}

Caso 1: Problema unidimensional, en estado estable, con fenómenos de conducción y convección [5]

El problema consiste en determinar la temperatura interior y exterior de una lámina de vidrio, siendo conocidas las temperaturas del ambiente interior y exterior de la lámina, así como los coeficientes peliculares respectivos y la conductividad térmica. La figura 1 ilustra tanto la geometría del problema como los datos principales de éste.

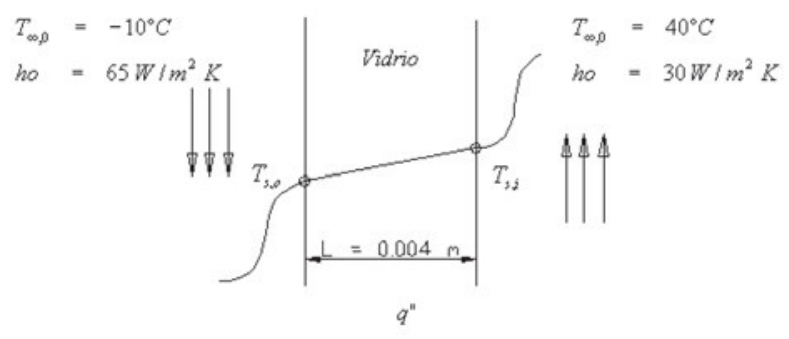

Fig. 1 Esquema del problema 1.

El criterio de selección de este problema se basó en la simplicidad del modelamiento geométrico y que considera dos mecanismos de transferencia de calor. La solución presentada en el texto es del tipo analítica.

Las subcompetencias o capacidades que se deseaban evaluar a través de la solución de este primer problema fueron:

- Grado de aprendizaje del software.

- Modelar de problemas con elementos de tipo lineal.

- Seleccionar los tipos de elementos adecuados al problema.

- Transferir adecuadamente los datos del problema al modelo.

- Obtener, interpretar y analizar los resultados que entrega el software.

- Replantear la estrategia de solución, en caso de que el modelo desarrollado no entregue los valores numéricos correctos.
Resultados alcanzados:

- El alumno logró modelar con éxito este primer problema, ya que los resultados obtenidos por él difieren en el orden del $1 \%$ con respecto a los que reporta el texto.

- La evaluación mostró que el alumno alcanzó las capacidades propuestas.

Las figuras 2, 3 y 4 muestran el modelo planteado por el estudiante y la visualización de los resultados que entrega el software.

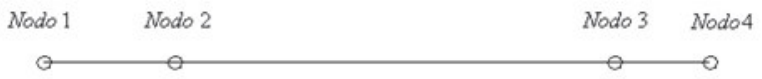

Fig. 2 Modelo de elementos finitos.

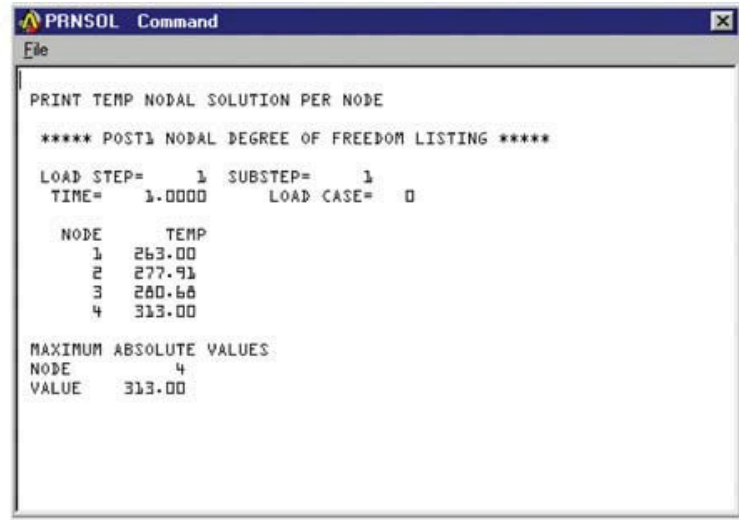

Fig. 3 Resultado de temperatura en los nodos

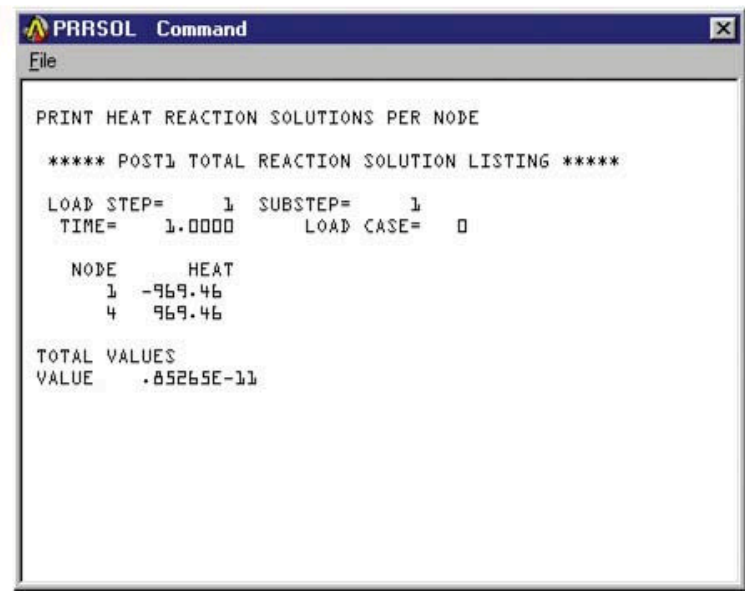

Fig. 4 Flujo de calor a través del cuerpo.

Caso 2: Conducción bidimensional con generación interna de calor [6]

Este problema trata sobre la determinación de las temperaturas de un alambre, que presenta generación 
interna de calor. Se conoce, además, la temperatura de la superficie y la conductividad térmica del material.

La figura 5 ilustra el problema.

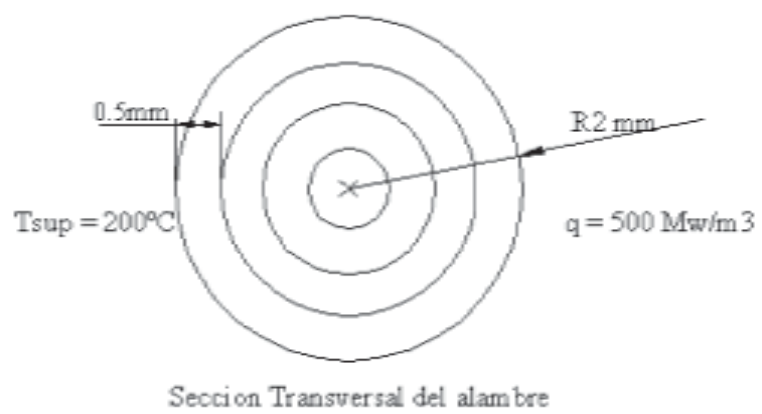

Fig. 5 Esquema del problema 2.

Este problema presenta un mayor grado de complejidad, ya que se debe decidir la forma de discretizar el modelo. Esto implica que el estudiante ya debe aplicar criterio técnico. En cuanto a la forma de solución presentada en el texto, ésta es realizada mediante el método de las diferencias finitas.

Las capacidades que se deseaban evaluar a través de la solución de este problema fueron:

- Modelar un problema utilizando condiciones de simetría.

- Definir la geometría del modelo utilizando el tipo de sistema coordenado más conveniente.

- Seleccionar el tipo de elemento adecuado y definición de los mismos.

- Evaluar la capacidad de decisión en cuanto a cómo y dónde se deben aplicar las cargas térmicas y restricciones al modelo.

- Solucionar, analizar y validar los resultados.

Resultados:

El estudiante logró, después de algunos intentos, encontrar un modelo adecuado y obtener los resultados correctos. Esto permite afirmar que su capacidad de análisis para abordar el problema e interacción con el software para solucionar un problema más complejo se vio incrementada.

Las figuras 6, 7 y 8 muestran la estrategia utilizada por el estudiante y resultados obtenidos.

El modelo que utilizó el estudiante es un sector circular, ya que consideró la condición de simetría geométrica del cuerpo.

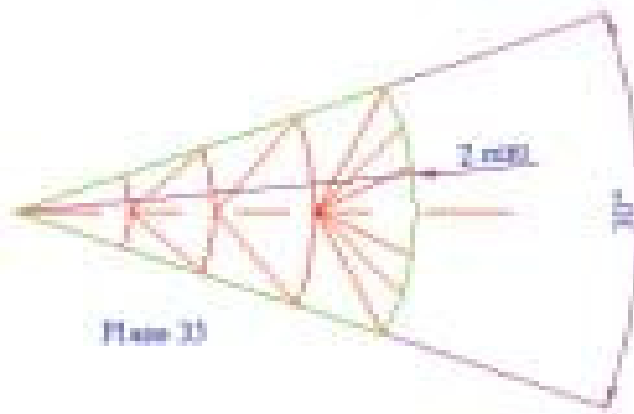

Fig. 6 Geometría del modelo.

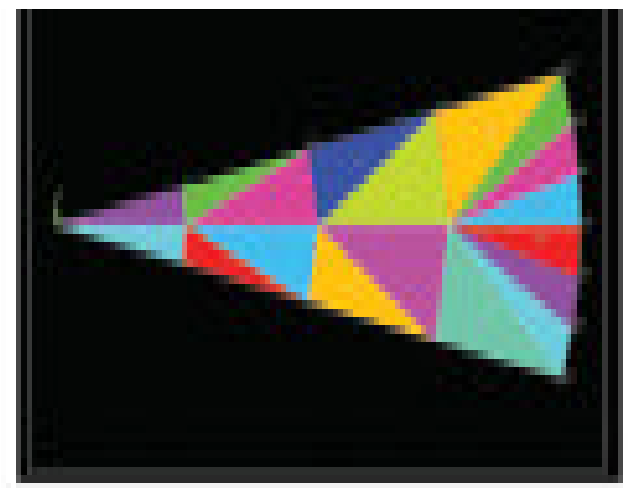

Fig. 7 Modelo a utilizar.

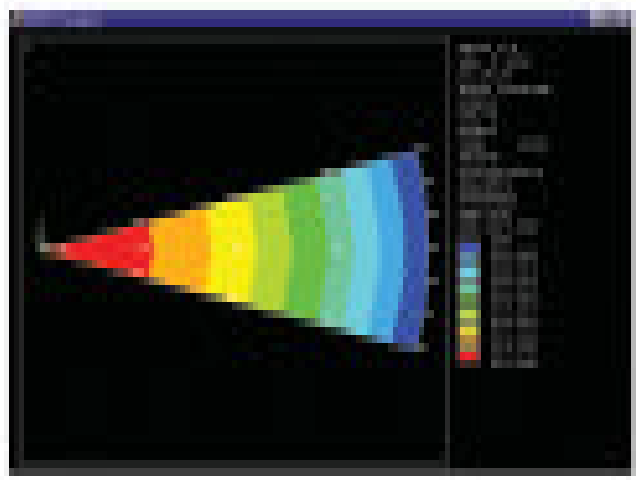

Fig. 8 Modelo con los resultados en forma gráfica.

\section{Caso 3: Conducción de calor a través de aletas rectangulares [7]}

Este problema consiste en determinar el flujo de calor y la temperatura en el extremo de una varilla de sección rectangular de geometría conocida, así como los valores de la conductividad térmica del material, el coeficiente pelicular convectivo entre la varilla y el fluido, las temperaturas del fluido medio y de la base de la aleta.

La figura 9 presenta esquemáticamente el problema, el que es solucionado en el texto mediante la teoría de aletas. 


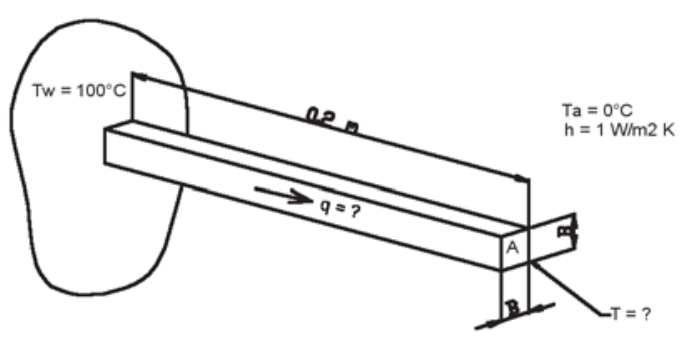

Fig. 9 Esquema del problema 3.

El criterio de selección de este problema se basó en que éste presenta mayores dificultades en la formulación de una estrategia de solución. De manera que las subcompetencias que se deseaban evaluar a través de la solución de este problema fueron, en términos prácticos, las mismas que en el caso anterior, esto es:

- Analizar el problema y definir el modelo a utilizar.

- Modelar la geometría.

- Seleccionar el tipo de elemento más adecuado para el tipo de problema.

- Aplicar los parámetros que definen el problema.

- Analizar la forma de aplicar las cargas térmicas.

- Obtener una solución, analizarla e interpretarla.

Las figuras 10,11 y 12 muestran la estrategia desarrollada por el estudiante y resultados obtenidos de su aplicación.

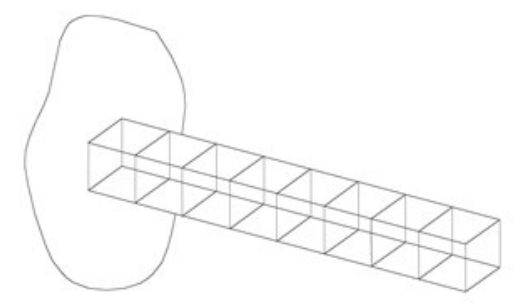

Fig. 10 Esquema del modelo de elementos finitos utilizado.

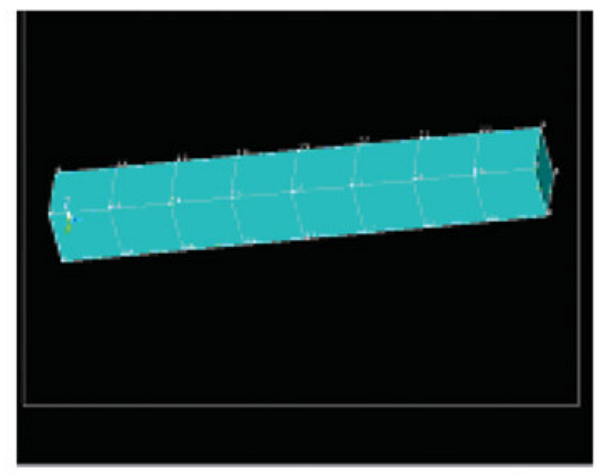

Fig. 11 Modelo de elementos finitos creado.

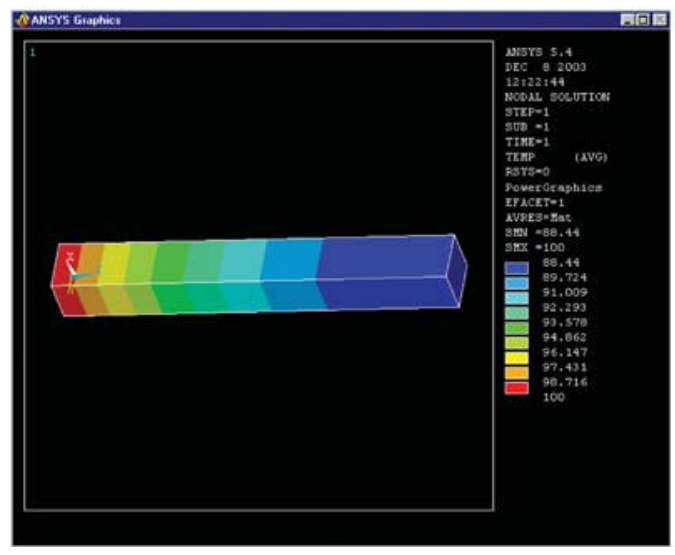

Fig. 12 Ventana gráfica con los resultados.

Resultados:

El estudiante pudo nuevamente modelar con éxito el problema y llegar a los resultados indicados en el texto, con una mayor velocidad y eficacia que en los dos casos anteriores.

\section{Caso 4. Conducción de calor en una placa [6]}

El problema trata sobre una placa de cerámica que está en contacto con un material sólido de alta conductividad térmica, de tal manera que se mantiene a una temperatura constante. Las condiciones de borde corresponden a la superficie inferior de la cerámica aislada y la superficie superior está sujeta a convección y radiación.

La figura 13 presenta esquemáticamente el problema, que es solucionado en el texto mediante la aplicación del método de diferencias finitas.

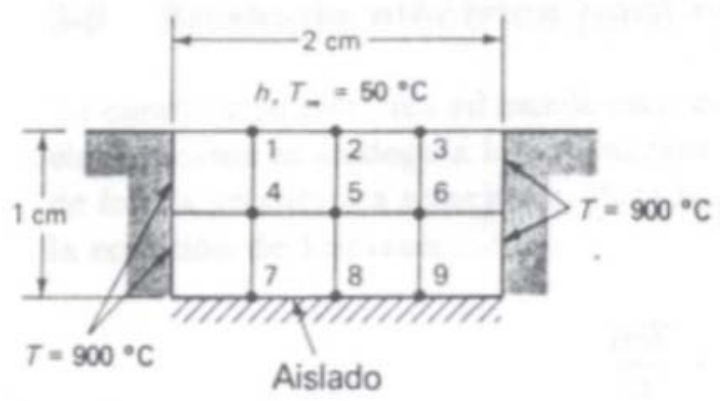

Fig. 13 Esquema del problema 4.

Este problema se seleccionó, dado que se aumenta notoriamente la complejidad de la solución, ya que se deben modelar los tres tipos cargas térmicas que actúan y seleccionar tipos de elementos más complejos. 
Las capacidades que se buscaban evaluar mediante la solución de este problema fueron:

- Abordar y resolver un problema más complejo, considerando que ya posee una experiencia en el uso del software.

- Caracterizar, utilizando los elementos correspondientes, los mecanismos de convección y radiación, los que se presentan simultáneamente en la superficie de la placa.

- Evaluar la capacidad de análisis relativo a cómo aplicar las constantes y propiedades en la solución del problema.

- Distinguir cómo y dónde aplicar los mecanismos de conducción, convección y radiación.

- Obtener una solución, analizarla e interpretar los resultados.

La figura 14 muestra el tipo de modelamiento y los tipos de elementos que empleó el estudiante para plantear una solución del problema.

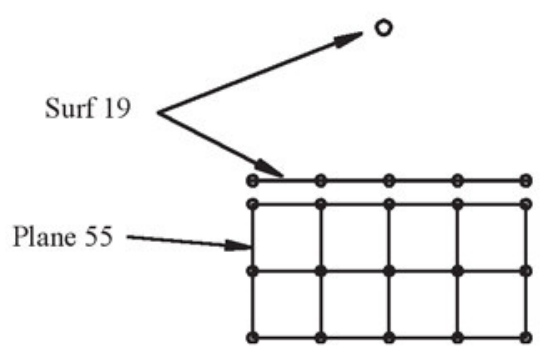

Fig. 14 Esquema del modelo utilizado.

La figura 15 muestra la solución en términos gráficos alcanzada por el estudiante, lo que implicó que éste estableciera el modelo geométrico, estableciera su sistema de coordenadas y su discretización, considerando un nodo libre para incluir la radiación.

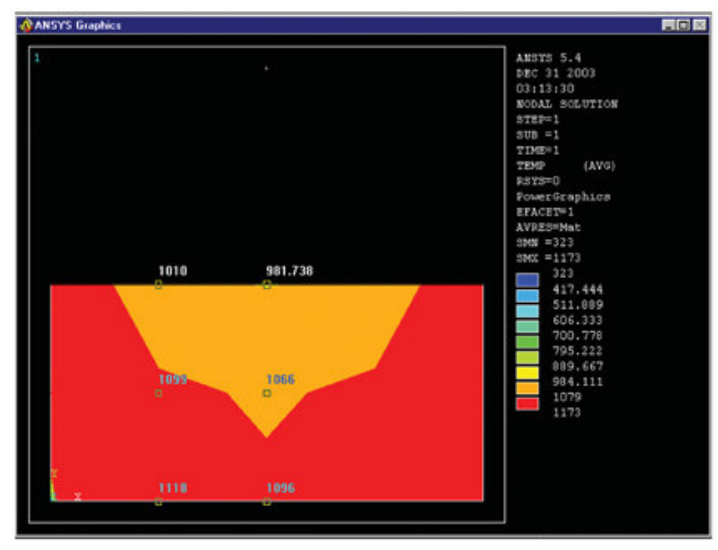

Fig. 15 Ventana gráfica con los resultados de distribución de temperatura.
Resultados:

El estudiante pudo abordar y dar solución a este problema más complejo y los resultados alcanzados difieren en un rango entre 0,2 y un $1 \%$, de los que entrega el texto. En este caso, el estudiante mostró un avance notable en su capacidad para el planteamiento de una estrategia de solución.

\section{Problema 5. Conducción de calor transiente a través de un cuerpo tridimensional sujeto a convección [5]}

Este problema consiste en determinar las temperaturas en dos puntos específicos de un cilindro sólido, tras un cierto intervalo de tiempo. La solución desarrollada en el texto es por medio del método de las diferencias finitas La figura 13 presenta esquemáticamente el problema.

Las condiciones de borde de este problema son de dos tipos. El primero corresponde a las condiciones de borde espaciales y relaciona la temperatura inicial del cuerpo y la existencia de convección en las distintas superficies del cilindro como condición de borde.

El segundo tipo de condiciones de borde son las temporales, ya que el problema no es estacionario, esto implica que se deben considerar cuatro coordenadas, tres espaciales y una temporal, lo que constituye una dificultad importante del problema.

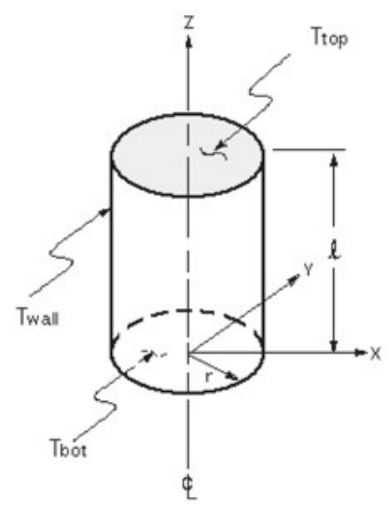

Fig. 16 Esquema del problema 5.

Las razones para seleccionar este problema fueron de cierta manera enunciadas en los párrafos anteriores, ya que el grado de dificultad del problema es considerable, tanto por el aspecto de tridimensionalidad geométrica como por la condición de temporalidad.

Las capacidades que se buscaban evaluar mediante la solución de este problema fueron: 
- Abordar y solucionar un problema de complejidad mayor a los que ya había resuelto.

- Modelar el problema, aprovechando las condiciones de simetría del problema.

- Seleccionar los tipos de elementos adecuados para la solución de problemas de tipo transiente.

- Ingresar al software tanto los datos del problema como las restricciones.

- Obtener una solución, analizarla e interpretarla a partir de los resultados que entrega el software.

La figura 17 muestra el tipo de elemento seleccionado por el estudiante, que corresponde a un elemento tridimensional prismático con capacidad de conducción térmica, que es aplicable tanto a problemas de tipo estacionario como transiente.

Como datos para alimentar el software, se utilizaron la geometría del cuerpo, las propiedades del material, tales como: su densidad, su coeficiente de expansión térmica, su conductividad térmica y el calor específico. A lo que se sumaron las condiciones de borde representadas por coeficiente pelicular convectivo y la temperatura del fluido. Además, de la temperatura inicial del cuerpo y el intervalo de tiempo considerado.

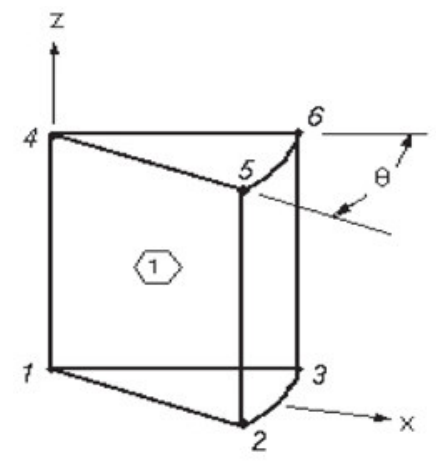

Fig. 17 Esquema del tipo de elemento empleado.

La figura 18 presenta al modelo con el estado de cargas elaborado por el estudiante.

La figura 19 muestra la solución en forma gráfica que alcanzó el estudiante.

Resultados:

El estudiante no sólo pudo abordar y alcanzar una solución satisfactoria a este problema, sino que su resultado mostró una diferencia de un $10 \%$, respecto del resultado indicado en el texto. Por lo cual, procedió a refinar la malla, en el evento que fuese un problema asociado a ésta. Sin embargo, los resultados alcanzados no se aproximaron a la solución propuesta. La razón de esta situación se debe a que el estudiante alcanzó una precisión mayor en su solución, respecto a la planteada en el texto.

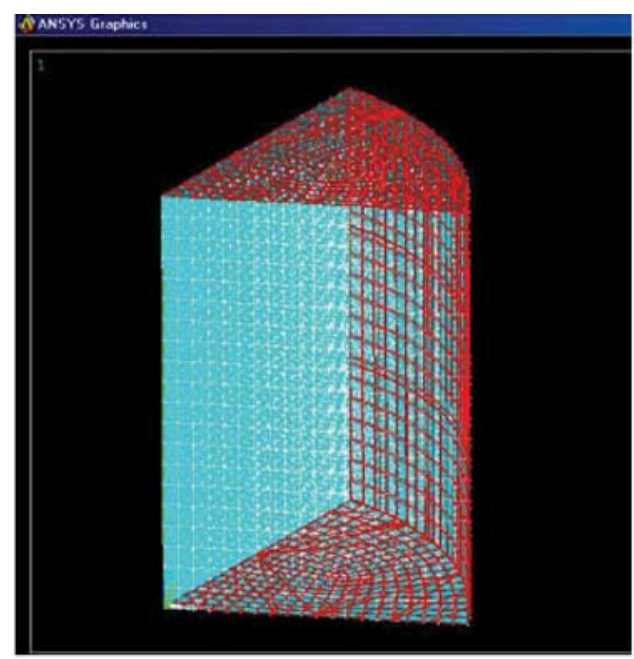

Fig. 18 Modelo creado, con las cargas aplicadas.

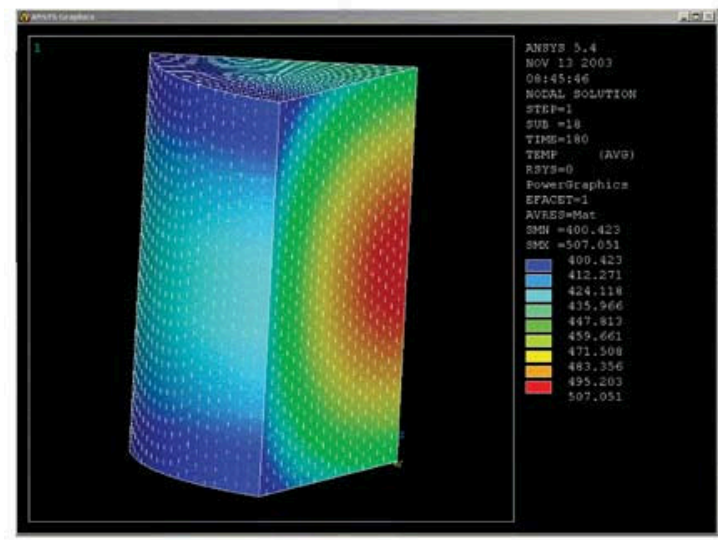

Fig.19 Ventana con los resultados en forma gráfica.

Ante esta diferencia de valores asociada a la solución del problema, el estudiante se mostró inicialmente desconcertado, pero tras refinar la malla indicó con seguridad que su resultado era correcto y que la diferencia se debía sólo al método se solución empleado o a un problema de impresión tipográfica del texto. Esto denotó la seguridad y competencia adquirida por el estudiante tras el proceso de enseñanza-aprendizajeevaluación.

\section{CONCLUSIONES}

Este trabajo permitió apreciar los logros alcanzados por un estudiante de bajo rendimiento académico, ante una distinta metodología de enseñanza-aprendizajeevaluación, basada en el autoaprendizaje y el trabajo autónomo de un tema específico de ingeniería que no se considera dentro del programa de estudio de su carrera. 
Los resultados cognitivos y procedimentales alcanzados por el estudiante son sorprendentes, si se considera que la calificación que alcanzó su trabajo escrito fue 6,3 , mientras que la presentación y defensa de su trabajo fue evaluada con nota 6,8. Esto conduce a considerar la necesidad de desarrollar nuevas experiencias e investigaciones en torno a los factores que hacen que un estudiante no tenga un buen rendimiento académico en los cursos formales, así como en el desarrollo de estrategias educativas tendientes a mejorar esta situación.

Respecto a la metodología utilizada, se pudo constatar que ésta fue adecuada para el perfil del estudiante, ya que éste al final del trabajo fue capaz de:

- Comprender aspectos básicos del método de los elementos finitos.

- Comprender la problemática que existe para abordar la solución de problemas mediante el método de los elementos finitos.

- Alcanzar un manejo satisfactorio del software y alcanzar las competencias necesarias para resolver problemas típicos de transferencia de calor.

- Realizar un documento escrito donde desarrolla la estrategia que empleó para enfrentar los problemas y para que sirva de guía de autoaprendizaje a otros estudiantes.

Finalmente, es posible concluir que la utilización de las técnicas de autoaprendizaje, en el proceso de enseñanzaaprendizaje-evaluación en ingeniería, son una buena alternativa de estrategia a seguir, en el caso de iniciar el aprendizaje del método de elementos finitos, ya que es muy atractiva para el estudiante, a la vez que se logra fácilmente motivarlo, al tiempo que se refuerzan su autoestima y los conocimientos ya adquiridos.

\section{REFERENCIAS}

[1] J. Ginés. "La necesidad del cambio educativo para la sociedad del conocimiento". Revista Iberoamericana de Educación No 35. 2004.

[2] W. Salas. "Formación por competencias en educación superior". Revista Iberoamericana de Educación. N 36/9. 2005.

[3] A. Carretero. "Metodología didáctica para la enseñanza de geometría descriptiva basada en un tutor-evaluador y en generador de ejercicios integrados en un entorno de propósito constructivo general". Tesis Doctoral. Universidad Politécnica de Madrid. 2001

[4] H. Pardo. "Rutas de autoaprendizaje y contenidos en espacios interactivos. ¿La nueva pedagogía?”. Revista mexicana de comunicación. No 93. 2005.

[5] F. Incropera, D. Dewitt. "Fundamentos de transferencia de calor". 4 ${ }^{\mathrm{a}}$ Ed. Prentice Hall., pp. 134. Prob. 3.2 y pp. 249. Ejemplo 5-6. 1989.

[6] J. Holman. "Transferencia de calor". $7^{\mathrm{a}}$ Ed. CECSA, pp. 109. Ejemplo 3-5, pp. 116. Ejemplo 3-8. 1996.

[7] J. Manrique. "Transferencia de calor". Editorial Harla. 1987. 